\title{
Thylakoid-integrated recombinant Hcf106 participates in the chloroplast Twin Arginine Transport (cpTat) system
}

\author{
Qianqian Ma ${ }^{\mathrm{a}, 1}$, Kristen Fite ${ }^{\mathrm{b}, 2}$, Christopher Paul New ${ }^{\mathrm{a}}$, and Carole Dabney-Smith ${ }^{\mathrm{a}, \mathrm{b}^{*}}$ \\ ${ }^{a}$ Graduate Program in Cell, Molecular, and Structural Biology, Miami University, Oxford, \\ $\mathrm{OH}$

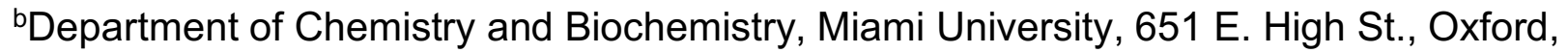 \\ $\mathrm{OH}$ 45056, USA \\ ${ }^{1}$ Current Address: Johns Hopkins University School of Medicine, Baltimore, MD. \\ ${ }^{2}$ Current Address: Wright State University, Boonshoft School of Medicine, Dayton, $\mathrm{OH}$. \\ ${ }^{*}$ Correspondence \\ Carole Dabney-Smith, Miami University, 651 E. High St., Oxford OH, 45056 \\ E-mail: adabney@miamioh.edu
}

\begin{abstract}
The chloroplast Twin arginine transport (cpTat) system distinguishes itself as a protein transport pathway by translocating fully-folded proteins, using the proton-motive force (PMF) as the sole source of energy. The cpTat pathway is evolutionarily conserved with the Tat pathway found in the plasma membrane of many prokaryotes. The cpTat (E. coli) system uses three proteins, Tha4 (TatA), Hcf106 (TatB), and cpTatC (TatC), to form a transient translocase allowing the passage of precursor proteins. Briefly, $\mathrm{cpTatC}$ and Hcf106, with Tha4, form the initial receptor complex responsible for precursor protein recognition and binding in an energy-independent manner, while a separate pool of Tha4 assembles with the precursor-bound receptor complex in the presence the PMF. Analysis by blue-native polyacrylamide gel electrophoresis (BN-PAGE) shows that the receptor complex, in the absence of precursor, migrates near $700 \mathrm{kDa}$ and contains cpTatC and Hcf106 with little Tha4 remaining after detergent solubilization. To investigate the role that Hcf106 may play in receptor complex oligomerization and/or stability, systematic cysteine substitutions were made in positions from the $\mathrm{N}$-terminal transmembrane domain to the end of the predicted amphipathic helix of the protein. BNPAGE analysis allowed us to identify the locations of amino acids in Hcf106 that were critical for interacting with cpTatC. Oxidative cross-linking allowed us to map interactions of the transmembrane domain and amphipathic helix region of Hcf106. In addition, we showed that in vitro expressed, integrated Hcf106 can interact with the precursor signal peptide domain and imported $\mathrm{cpTatC}$, strongly suggesting that a subpopulation of the integrated Hcf106 is participating in competent cpTat complexes.
\end{abstract}

\section{Key words}

Twin arginine transport, cross-linking, truncation, receptor complex, oligomerization 


\section{Introduction}

The Iwin Arginine Transport system (Tat) is one of two protein transport pathways that deliver proteins to the lumen of the plant thylakoid; a homologous Tat pathway is also found in a wide range of bacteria (Celedon and Cline, 2013; Berks, 2015; Cline, 2015). The Tat system is distinguished from other protein transport pathways, such as the well-characterized Secretory (Sec) system, by transporting fullyfolded precursor proteins using only the proton-motive force (PMF) for energy (Cline and Mori, 2001; Braun et al., 2007; Gérard and Cline, 2007). Proteins transported by the Tat pathway are usually cytosolically synthesized as higher molecular weight precursors containing cleavable $\mathrm{N}$-terminal signal peptides with an obligate twin arginine motif (RR) as is reflected in the name of the system. Tat systems can transport precursor proteins of different sizes ranging from $2 \mathrm{kDa}$ to over $100 \mathrm{kDa}$, as well as substrates that form oligomers (Ma and Cline, 2010; Celedon and Cline, 2012). A recent stoichiometry study suggested that each individual Tat translocase can bind up to eight precursor proteins when fully saturated and is transport active when sufficient Tha4 is present (Celedon and Cline, 2012). However, mechanistic detail of how the receptor complex organizes to accommodate the transport of multiple precursor proteins in concert is an important question that needs to be addressed.

In thylakoid (as well as in E. coli) three membrane-bound protein components, Tha4 (TatA), Hcf106 (TatB), and cpTatC (TatC), are responsible for the twin arginine dependent translocation of precursor cargo (Cline and Mori, 2001; Celedon and Cline, 2013). Current models of the Tat system suggest a cyclical mechanism in which the receptor complex is comprised of $\mathrm{cpTatC}$ and Hcf106 tightly bound to each other with a loosely associated Tha4 that serves as the initial site of precursor recognition and binding, followed by assembly of additional Tha4 homo-oligomers, which are proposed to form the translocation pore (Bolhuis et al., 2001; Cline and Mori, 2001; Dabney-Smith et al., 2006; Dabney-Smith and Cline, 2009; Aldridge et al., 2012; Pal et al., 2013). Hcf106 is structurally similar to Tha4 in that both contain an amino terminal transmembrane domain (TMD), followed by a hinge region, an amphipathic $\alpha$-helix $(\mathrm{APH})$, and a loosely structured carboxyl terminus (C-tail). Recently, the structures of Tha4 and Hcf106 bacterial homologs, TatA and TatB, have been solved which agree with previous predictions (Hu et al., 2010; Zhang et al., 2014) and even more recently the structure to Tha4 from Arabidopsis thaliana (Pettersson et al., 2018). Despite the sequence similarity with Tha4, the two proteins are not interchangeable and thus appear to have distinct functions (Dabney-Smith et al., 2003).

Although the cpTat receptor complex has been well studied for its essential role in recognizing precursor proteins, few studies in plants have addressed the role of Hcf106 in this process, particularly, how each individual Hcf106 is organized in the multimeric receptor complex as compared to the bacterial homolog TatB (Bolhuis et al., 2001; Alami et al., 2003; Lee et al., 2006; Holzapfel et al., 2007; Rollauer et al., 2012; Behrendt and Bruser, 2014). The aim of the present work was to establish a method allowing the exploration Hcf106 organization using systematic cysteine substitution. As proof of principle, we have used this method to map Hcf106-Hcf106 interactions in thylakoid membranes.

Previous studies showed that in vitro translated Hcf106 can integrate into thylakoid in a manner presumably similar to endogenous Hcf106 and exists in a 
receptor complex with cpTatC (Gérard and Cline, 2006), which was capable of binding precursor proteins (Mori and Cline, 2002; Gérard and Cline, 2006). What remains unclear from the results of these studies is whether the integrated Hcf106 were simply members of the complex or directly participated in binding either precursor and/or $\mathrm{cpTatC}$. Here, we have used cysteine scanning and disulfide bond formation to systematically map Hcf106 interactions through the TMD to the APH regions, which are known to be of great importance to the organization of the receptor complex. We observed that single cysteine-substituted Hcf106 protomers integrate into isolated thylakoid, that most variants are resistant to alkaline extraction. And that they localize in a $700 \mathrm{kDa}$ complex by blue-native PAGE, suggesting that they are fully integrated into the membrane. Interaction sites of Hcf106-Hcf106 were obtained using copper (II)-1, 10-phenanthroline (CuP)-induced cross-linking which provided vital clues for the organization of Hcf106. Using double cysteine substitution in Hcf106, we could detect an Hcf106 oligomer as large as an octamer but could not distinguish if these oligomers were in the receptor complex or part of a separate pool of Hcf106. However, integrated Hcf106 was capable of interacting with transport competent precursor in a specific manner and with exogenous, imported cpTatC. From these data we conclude that integrated Hcf106 is capable of associating with and participating in the function of the cpTat translocase.

\section{Materials and Methods}

\subsection{Preparation of chloroplasts and thylakoid membranes}

Intact chloroplasts were prepared from 10-12 day-old pea seedlings (Pisum sativum L. cv. Laxton's Progress 9 or Little Marvel) as described (Cline et al., 1993). Intact, isolated chloroplasts were suspended to $1 \mathrm{mg} / \mathrm{ml}$ chlorophyll in import buffer (IB, $50 \mathrm{mM}$ HEPES-KOH, pH 8.0, $330 \mathrm{mM}$ sorbitol) and kept on ice until used. Isolated thylakoid were obtained by osmotic lysis of intact chloroplasts. Briefly, intact chloroplast suspensions were pelleted for $5 \mathrm{~min}$ at $1000 \mathrm{xg}$, supernatant removed, and suspended at $1 \mathrm{mg} / \mathrm{ml}$ chlorophyll in lysis buffer (50 mM HEPES-KOH, pH 8.0, $10 \mathrm{mM} \mathrm{MgCl}_{2}$ ) with incubation on ice for $5 \mathrm{~min}$. Following lysis, an equal volume of IB, $10 \mathrm{mM} \mathrm{MgCl} 2$ was added to the lysate, the thylakoid were then pelleted at $3200 \mathrm{xg}$ for $8 \mathrm{~min}$, and suspended at $1 \mathrm{mg} / \mathrm{ml}$ chlorophyll in IB, $10 \mathrm{mM} \mathrm{MgCl}_{2}$ (Aldridge et al., 2012). For single Cys interaction studies, thylakoid were suspended in $50 \mathrm{mM} \mathrm{N}$-ethylmaleimide (NEM) in $\mathrm{IB}, 10 \mathrm{mM} \mathrm{MgCl}$ and incubated on ice for $10 \mathrm{~min}$ to prevent non-specific crosslinking from endogenous free sulfhydral groups. NEM-treated thylakoid were subsequently pelleted and washed with 3 volumes of IB, $10 \mathrm{mM} \mathrm{MgCl}_{2}$ before use.

\subsection{Generation of Cysteine-substituted mature Hcf106 and C-tail truncation Hcf1061-107}

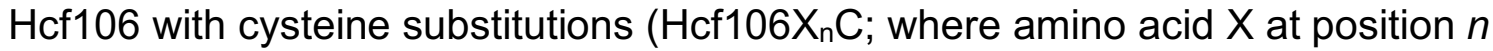
was replaced by cysteine) were generated by QuikChange mutagenesis (Agilent Technologies) according to manufacturer's instructions. The template used for mutagenesis was the coding sequence for mature Hcf106 (lacking the targeting peptide) in the plasmid pGEM-4Z. The coding sequence for Hcf106 begins with MASLFGVGAPEA.... Cloned constructs were verified by DNA sequencing on both strands at the Center for Bioinformatics and Functional Genomics at Miami University. 
135 For the C-tail truncation of $\mathrm{Hcf106}_{1-107}$, internal stop codons were inserted via primer-

136 based mutagenesis to generate the C-deletion of 69 amino acids. The size of the

137 truncated Hcf106 is about $17 \mathrm{kD}$ by SDS-PAGE analysis.

\subsection{Preparation of radiolabeled recombinant Hcf106 proteins}

Radiolabeled Hcf106 variants were prepared by in vitro translation in a wheat

\subsection{In vitro integration assay}

In vitro translated $\left[{ }^{3} \mathrm{H}\right] \mathrm{Hcf} 106 \mathrm{X}_{\mathrm{n}} \mathrm{C}$ was integrated into NEM-pretreated thylakoid (100 $\mathrm{\mu g}$ chlorophyll equivalent) for $25 \mathrm{~min}$ at $25^{\circ} \mathrm{C}$. For Hcf106 $1-107$, in vitro translated protein was directly integrated into isolated thylakoid. Reactions were terminated by transfer to $0^{\circ} \mathrm{C}$ and thylakoid were recovered by centrifugation at $3200 \mathrm{xg}$ for $8 \mathrm{~min}$. Recovered thylakoid were washed once with 2 volumes of IB, $10 \mathrm{mM} \mathrm{MgCl} 2$.

\subsection{Alkaline extraction assay}

Thylakoid with Hcf106 integrated were suspended to $1 \mathrm{~mL}$ with $0.2 \mathrm{M} \mathrm{Na}_{2} \mathrm{CO}_{3}$ or $0.1 \mathrm{M} \mathrm{NaOH}$ and incubated for $60 \mathrm{~min}$ on ice. Thylakoid were then recovered by centrifugation at $100,000 \mathrm{xg}$ for $15 \mathrm{~min}$. Pellets were suspended in $30 \mu \mathrm{l}$ of $20 \mathrm{mM}$ EDTA $1 \times$ IB and mixed with the same volume of $2 \times$ reducing sample solubilizing buffer ( $2 \times$ SSB (red); $100 \mathrm{mM}$ Tris-HCl (pH 6.8), $0.2 \mathrm{M} \mathrm{DTT}, 5 \%$ SDS, and $30 \%$ glycerol). Samples were analyzed by SDS-PAGE and fluorography.

\subsection{Blue-native gel electrophoresis}

Hcf106 integrated thylakoid $(\sim 1 \mathrm{mg} / \mathrm{ml}$ chlorophyll) were dissolved in $2 \%$ digitonin with end-over-end mixing for $1 \mathrm{~h}$ at $4{ }^{\circ} \mathrm{C}$ and centrifuged at $100,000 \mathrm{xg}$ for $30 \mathrm{~min}$. Supernatant was mixed with 0.1 vol $10 \times$ BN-PAGE sample buffer $(5 \%$ Serva G, $30 \%$ sucrose in $100 \mathrm{mM}$ BisTris- $\mathrm{HCl}, 500 \mathrm{mM}$ 6-amino-caproic acid, $\mathrm{pH}$ 7.0) as described by Cline and Mori (Cline and Mori, 2001). Gels were analyzed by fluorography or subjected to immunoblotting as described (Cline and Mori, 2001). Molecular markers used for blue native gels were dimeric and monomeric ferritin (880 kDa and $440 \mathrm{kDa}$, respectively) and bovine serum albumin (BSA) dimer (132 kDa).

\subsection{Oxidative cross-linking by disulfide bond formation}

Hcf106 integrated thylakoid were used for cross-linking reactions. $1 \mathrm{mM}$ copper (II)-1, 10-phenanthroline (CuP from a $150 \mathrm{mM}$ stock) was added as an oxidant to catalyze disulfide formation between proximal cysteine residues. The CuP stock solution $(150 \mathrm{mM})$ contained $150 \mathrm{mM} \mathrm{CuSO}_{4}$ and $500 \mathrm{mM} \mathrm{1,} \mathrm{10-phenanthroline}$ (Dabney-Smith et al., 2006). Cross-linking reactions were carried out for 5 min before stopping with $50 \mathrm{mM}$ ethylmaleimide (NEM, from a $1 \mathrm{M}$ stock in ethanol) and diluted two fold with $1 \times \mathrm{IB}, 10 \mathrm{mM} \mathrm{MgCl}$. Thylakoids were recovered by centrifugation at $3200 \mathrm{xg}$ for $8 \mathrm{~min}$, the supernatants removed, and the pellet was suspended in $1 \times \mathrm{IB}, 5 \mathrm{mM}$ EDTA, $10 \mathrm{mM}$ NEM and subjected to centrifugation and supernatant removal. Thylakoid 
pellets were suspended to $\sim 1 \mathrm{mg} / \mathrm{ml}$ chlorophyll and divided into two, centrifuged at $3200 \mathrm{xg}$ for $8 \mathrm{~min}$, and the supernatants removed. The first half was suspended in $2 \times$ non-reducing sample solubilizing buffer (2x SSB (nr); $100 \mathrm{mM}$ Tris-HCl (pH 6.8), $8 \mathrm{M}$ urea, $5 \%$ SDS, and $30 \%$ glycerol) and the second half was suspended in $2 \times$ SSB (red). Samples then were analyzed by SDS-PAGE and fluorography. The intensities of the bands were quantified with ImageJ software (Schneider et al., 2012).

\subsection{Disulfide cross-linking of imported cpTatC and integrated Hcf106 \\ The precursor to cpTatC, pre-cpTatCaaaV270C (Kenneth Cline, University of} Florida), was used as a source of exogenously integrated cpTatC according to published methods (Aldridge et al., 2014). Three native cysteines were substituted with alanine, hence the 'aaa' designation, and a single cysteine substitution was added at position V270. Radiolabeled in vitro translated pre-cpTatCaaaV270C was incubated with chloroplasts $(0.33 \mathrm{mg} / \mathrm{ml}$ chlorophyll $)$ and $5 \mathrm{mM} \mathrm{Mg}$-ATP in IB with $100 \mu \mathrm{E} / \mathrm{m}^{2} / \mathrm{s}$ of white light in a $25^{\circ} \mathrm{C}$ water bath for $40 \mathrm{~min}$. After import, intact chloroplasts were treated with thermolysin for 40 min at $4^{\circ} \mathrm{C}$ and isolated by centrifugation through a $35 \%$ Percoll (GE Healthcare) cushion in IB, 5 mM EDTA and washed with IB (Cline et al., 1993). Thylakoid membranes were prepared from isolated chloroplasts by osmotic lysis and centrifugation as described above and suspended in IB, $5 \mathrm{mM} \mathrm{MgCl}_{2}$ to $\sim 1 \mathrm{mg} / \mathrm{ml}$ chlorophyll. In vitro translated, unlabeled Hcf106 was integrated into thylakoid as described above. Thylakoids were centrifuged at $3200 \mathrm{xg}, 8 \mathrm{~min}$ to remove unincorporated Hcf106 and washed with IB, $10 \mathrm{mM} \mathrm{MgCl}$. Samples were subjected to cross-linking as describe above.

\subsection{Disulfide cross-linking of integrated Hcf106 and precursor}

Radiolabeled, in vitro translated precursor tOE17-25C V-20F, containing an inserted cysteine 25 residues upstream from the signal peptide cleavage site, was incubated with thylakoids which had been pre-integrated with in vitro translated, unlabeled $\mathrm{Hcf} 106$ in IB, $10 \mathrm{mM} \mathrm{MgCl} 2$ at $100 \mu \mathrm{E} / \mathrm{m}^{2} / \mathrm{s}$ of white light in a $25^{\circ} \mathrm{C}$ water bath for 5 min. Samples were subjected to cross-linking as described above.

\subsection{Supplemental Information}

Supplemental Figure S1. Hcf106 with cysteine substitutions in the (A) N-terminus, (B) TMD, (C) hinge integrates into thylakoid and is resistant to alkaline extraction. Supplemental Figure S2. Quantification of Hcf106 dimer formation in the TMD and APH.

Supplemental Figure S3. Most Hcf106 dimers disappear in the presence of the reducing agent, dithiothreitol (DTT).

\section{Results}

3.1 Single cysteine variants of Hcf106 integrate into thylakoid membranes and are resistant to alkaline extraction

Earlier work demonstrated that in vitro translated wild type Hcf106, which lacks native cysteines, was able to spontaneously integrate into isolated thylakoid and was resistant to alkaline extraction by either $0.2 \mathrm{M}$ carbonate buffer $(\mathrm{pH} 9.5)$ or $0.1 \mathrm{M} \mathrm{NaOH}$ (pH 11.5) (Fincher et al., 2003). The $\mathrm{NaOH}$ treatment extracts proteins that are 
peripherally associated with membrane or are partially embedded into the membrane via a single transmembrane domain; whereas carbonate extraction is capable of stripping peripherally associated proteins and but not embedded proteins (Rolland et al., 2006).

We generated a series of single-cysteine substitutions from the predicted transmembrane domain to the end of the predicted amphipathic helix in Hcf106 (Figure 1) and investigated whether these cysteine substitutions, especially in the $\mathrm{TMH}$, affected the integration of Hcf106 into the thylakoid membrane as compared to wild type.

Wild type Hcf106 is largely resistant to alkaline extraction (Fincher et al., 2003). Likewise, each of the Cys-substituted variants of Hcf106 tested were able to integrate into isolated thylakoid and were resistant to $0.2 \mathrm{M}$ carbonate treatment. In addition, most of the variants were also resistant to $0.1 \mathrm{M} \mathrm{NaOH}$ extraction (Supplemental Figure $\mathrm{S} 1$ ); the exceptions are I15C, V17C, V18C, and L21C residues which are predicted to be in the hydrophobic core of the membrane. Thus, we concluded that the resistance of most single cysteine substituted Hcf106 to alkaline extraction indicates that the integration of recombinant Hcf106 into isolated thylakoids was not negatively affected.

\subsection{Hcf106 Cys-variants can be detected in the $700 \mathrm{kDa}$ receptor complex}

Blue-native polyacrylamide gel electrophoresis (BN-PAGE) has shown that the cpTatC/Hcf106 receptor complex migrates as a band at $\sim 700 \mathrm{kDa}$ after solubilization by the detergent digitonin (Cline and Mori, 2001; Fincher et al., 2003). In addition, previous studies showed that recombinant, wild type, thylakoid integrated Hcf106 also migrated at $700 \mathrm{kDa}$, as well as a separate pool of smaller complexes of $\sim 400 \mathrm{kDa}$ to $\sim 200 \mathrm{kDa}$ depending upon the detergent to membrane ratio (Mori et al., 2001; Fincher et al., 2003). Most of the single cysteine variants of Hcf106 were integrated into isolated thylakoid; therefore, we examined whether the integrated Cys variants would form oligomers when solubilized in detergent as shown by BN-PAGE (Figure 2). We reasoned that if the Cys variants of Hcf106 could interact with $\mathrm{cpTatC}$, then we would expect to find the Cys variants incorporated into a $\sim 700 \mathrm{kDa}$ complex when digitoninsolubilized thylakoid were analyzed by BN-PAGE.

We integrated the Hcf106 variants into isolated thylakoid and solubilized the membranes with digitonin followed with analysis by BN-PAGE. The ratio of detergent to thylakoid chlorophyll content was critical because previous studies demonstrated that an increase in the ratio of detergent to thylakoid resulted in the persistence of the 700 $\mathrm{kDa}$ receptor complex of $\mathrm{cpTatC/Hcf106}$ complex, while the Hcf106 homo-oligomeric complexes between $400 \mathrm{kDa}$ and $200 \mathrm{kDa}$ were disrupted (Cline and Mori, 2001; Fincher et al., 2003). We were most interested in the presence or incorporation of Hcf106 in the $700 \mathrm{kDa}$ receptor complex, so membrane solubilization was done at a ratio of $2 \%$ digitonin to $1 \mathrm{mg} / \mathrm{ml}$ chlorophyll to minimize the formation of the smaller homo-oligomeric complexes (Fincher et al., 2003). Bands seen at the bottom of the gel indicate the presence of smaller oligomers of Hcf106 (e.g., dimer to tetramer of 60-120 $\mathrm{kDa}$ ), but nothing in the $200-400 \mathrm{kDa}$ range. Of the cysteines placed close to the $\mathrm{N}$ terminus, such as G6C, V7C, G8C, A9C, P10C, and E11C, only G8C (Figure 2, lane 3) and $\mathrm{E} 11 \mathrm{C}$ (Figure 2, lane 6) were unable to migrate in a $700 \mathrm{kDa}$ complex but did integrate successfully into thylakoid (Supplementary Figure S1), suggesting that key 
contacts between Hcf106 and cpTatC were disrupted in those variants. Hcf106 variants with a Cys substitution in the transmembrane region such as A12C, L13C, V14C, I15C, G16C, V17C, V18C, A19C, L20C, L21C, and V22C (Figure 2, lanes 9-19), did migrate as a 700 kDa complex just like wild type Hcf106 (Figure 2, lanes 7-8, 21, 26, 39, 45) suggesting incorporation into the receptor complex. Most of the variants in the hinge region, i.e., F23C, G24C, K26C, or G27C (Figure 2, lanes 20, 22, 24, and 25) failed to incorporate into the receptor complex but were able to integrate into thylakoid (Supplemental Figure S1). P25C was able to incorporate into the receptor complex (Figure 2, lane 23). Cys variants in the APH region of Hcf106 showed a similar pattern to wild type when analyzed by BN-PAGE in that all single cysteine substitutions in this region did not abolish incorporation into the $700 \mathrm{kDa}$ complex (Figure 2, lanes 26-57). However, certain cysteine substitutions, for example, E30C, A32C, K37C, E41C, Q43C, and P44C (Figure 2, lanes 29, 31, 36, 41, 43, and 44), consistently showed a lower intensity at the $700 \mathrm{kDa}$ band, suggesting that cysteines in this region of the APH may negatively affect Hcf106 interaction with cpTatC (i.e., incorporation into the $700 \mathrm{kDa}$ complex), but did not affect the integration and membrane stability of the variant (Supplemental Figure S1).

\subsection{C-terminal of Hcf106 is dispensable for cpTatC-Hcf106 receptor complex formation}

Hcf106 contains a loosely-structured C-tail that was shown to not be required for receptor complex formation in the $E$. coli homolog, TatB (Maldonado et al., 2011). If the truncated Hcf106 could interact with $\mathrm{cpTatC}$, we reasoned that truncated protein could be used to demonstrate that the integrated Hcf106 is, in fact, incorporating into receptor complexes with endogenous cpTatC (Figure 3). We incubated increasing amounts of $\left[{ }^{3} \mathrm{H}\right] \mathrm{Hcf} 106_{1-107}$, lacking 69 amino acids from the $\mathrm{C}$ terminus, with isolated thylakoid and analyzed the membranes by BN-PAGE. As the concentration of in vitro translated $\left[{ }^{3} \mathrm{H}\right] \mathrm{Hcf106}{ }_{1-107}$ increased, two lower bands of $\sim 600 \mathrm{kDa}$ (Figure 3, lanes 2-4) and 500 $\mathrm{kDa}$ (Figure 3, lanes 3-4) appeared, suggesting that in vitro integrated $\left[{ }^{3} \mathrm{H}\right] \mathrm{Hcf} 106$ was competing with the endogenous Hcf106 for binding to endogenous cpTatC. The smaller complexes also contain cpTatC (Figure 3, lanes 9-12), indicating the C-tail does not play a critical role in receptor complex formation. Immunodetection of Hcf106 using the same thylakoid samples demonstrated that full length Hcf106 also migrated into smaller complexes due to the presence of the truncated variant. With these data, the insensitivity to alkaline extraction, and the incorporation into a $700 \mathrm{kDa}$ complex, we conclude that exogenously added Hcf106 is properly inserted into the thylakoid membrane, allowing us to use these Cys-substituted variants to probe the organization of Hcf106 in the receptor complex.

\subsection{The transmembrane domain and amphipathic helix regions of Hcf106 form self-contacts}

To characterize the organization of Hcf106-containing complexes, we looked at the organization of Hcf106 by studying interactions between neighboring Hcf106 proteins. We reasoned that interactions between Hcf106 proteins would indicate the organization of Hcf106 in the receptor complex by identifying sites specific for selfinteractions as well as provide insight into the organization of the separate pool of 
Hcf106. We took a cysteine scanning approach, which allowed us to map interactions between neighboring single cysteine substituted Hcf106 proteins or other cpTat components by formation of disulfide bonds between cysteines within $\sim 5 \AA$ of each. Hcf106 proteins containing single cysteine substitutions in the TMD or APH were integrated into isolated thylakoid. In the presence of an oxidant such as copper (II)-1,10phenanthroline (CuP), free cysteine sulfhydrals in close proximity will form stable disulfide bonds, which cause a mobility shift from $\sim 28 \mathrm{kDa}$ to $\sim 56 \mathrm{kDa}$ of the crosslinked proteins when analyzed by SDS-PAGE.

Residues close to the N-terminus of Hcf106 were in close proximity to the same residue of a neighboring Hcf106 (Figure 4, lanes 1-5) showing a significant amount of dimer formation, demonstrating that these amino acids are sufficiently close to form a disulfide bond or that this region is very flexible. On the other hand, when the Cys was placed in the TMD, i.e., E11C-L21C (Figure 4, lanes 6-16), dimer formation demonstrated a position-specific interaction, indicating a regular face of interaction in this portion of the transmembrane domain. As the cysteine substitution moved out of the TMD through the hinge and into the APH, i.e., V22C, F23C, and K26C (Figure 4, lanes 17, 18, 21) formed a dimer, whereas G24C, P25C, and G27C (Figure 4, lanes 19, 20, 336 22) did not.

The amphipathic helix of Hcf106 showed two different types of interaction. The $\mathrm{N}$-terminal proximal portion of the APH, i.e., L28C-P44 (Figure 4, lanes 23-39), showed no interaction overall, with the exceptions of L28C, L35C, E41C, and F42C (Figure 4, lanes $23,30,36,37)$, compared to the C-terminal proximal portion of the APH, T45CG65C (Figure 4, lanes 40-60), which demonstrated a stronger and position-specific cross-linking. For example, dimer was detectable for all Cys-substitutions in this segment, but dimers formed with cysteines at 149C or Q50C were more intense (Figure 4, compare lanes 44-45 with lanes 36-43) based on equal chlorophyll loading. The relative proportion of dimer to total protein was quantified by densitometric analysis (Supplemental Figure S3). The presence of a band at $56 \mathrm{kDa}$ was dependent upon disulfide formation because treatment of samples with the reducing agent, dithiothreitol (DTT), effectively depleted the dimer species. Interestingly, L21C and V22C were not reduced to the monomeric form (Supplemental Figure S3).

The results of the specific interactions on the Hcf106 TMD or APH are plotted on helical wheels (Figure 5). For example, a helical wheel projection of the TMD with the hinge region (residues A12C-G27C) emphasizes that Hcf106 self-interactions occur along no particular face (Figure 5A). The APH domain ( 40 amino acids) is too large to be clearly evaluated with one helical wheel, so we generated wheels for the $\mathrm{N}$-terminal half (i.e., L28-P44; (Figure 5B) and the C-terminal half (T45-G65; Figure 5C). The proline at position 44 was arbitrarily determined as the halfway point because it would serve to break the helix. The helical wheel of the $\mathrm{N}$-terminal portion of the APH shows a preferred face for Hcf106 self-interaction because L28, L35 and F42 fall along the hydrophobic face of the helix (Figure 5B), although we do see interactions at E41C and P44C, which do not fall on the same face of the predicted helix. However, we envision the APH could be very mobile or dynamic in the membrane, which may explain the interactions at E41C and P44C. Other residues, e.g., A29C-T38C, showed no selfinteractions indicating that these faces of the helix may be buried in the membrane, interacting with other cpTat components, or positioned such that they are not near each 
other on neighboring helices. The helical wheel of the C-terminal portion of the APH demonstrated that roughly three of four faces can interact with neighboring Hcf106 (Figure 5C), indicating that this portion of the helix might be flexible or that it could emanate from the membrane similarly to what was seen with Tha4 (Aldridge et al., 2012).

\subsection{Double-cysteine substitutions suggest a high-ordered Hcf106 complex}

Single cysteine substitutions can only detect dimers. To investigate the formation of higher-ordered oligomers, we constructed double cysteine substituted Hcf106 to detect an ability to form higher ordered protomers. We substituted two cysteines in the transmembrane helix of Hcf106, e.g., L13CL21C and V14CL20C, and the resulting proteins were integrated into thylakoid followed by oxidative crosslinking. Initially, when compared to the single cysteine variant L21C, the double Cys-substituted proteins L13CL21C and V14CL20C showed roughly half the integration into thylakoid as L21C (Figure 6A, compare lanes 7, 10 to 9,12), while double cysteine variants in the APH, e.g., I49CE61C and R54CL60C (Figure 6A, compare lanes 8, 11 to lanes 9, 12), were comparable to the amount of L21C integrated. Decreased integration of the TMD double Cys variants was likely due to the introduction of two cysteines into the helix, decreasing the hydrophobicity of the TMD. Unexpectedly, the cross-linking of these four cysteine variants did not show obvious Hcf106 multimers (Figure 6A, lanes 1-2, 4-5). Furthermore, when the V14CL20C variant was compared to its single cysteine parent, V14C, the double Cys variant also showed about $30 \%$ less integration into thylakoids (Figure 6B, lanes 1-2). However, when one cysteine was placed in the $\mathrm{N}$ terminus and the other in the TMD, e.g., G6CV14C, we observed Hcf106 multimers as high as octamers when analyzed by $10-20 \%$ acrylamide gradient gel PAGE (Figure 6, lanes 34).

To further investigate if the cross-linked Hcf106 multimers are part of the 700 kDa complex, we subjected the cross-linked G6CV14C double Cys variant to BN-PAGE (Figure 6C). Different cross-linking conditions were used including pre-treatment of the translation product with NEM to block the free cysteine (Figure 6, lane 1), the presence or absence of CuP (Figure 6, lanes 2 and 3), cross-linking without quenching (Figure 6, lane 4), and prolonged cross-linking (30 min) (Figure 6, lane 5). Samples were also subjected to non-reducing SDS-PAGE to monitor the status of cross-linking (Figure 6, lane 7-11). On BN-PAGE, we observed a significant amount of $700 \mathrm{kDa}$ complex when the double cysteine variant was cross-linked (Figure 6, lane 2-5). Additional bands were also observed (above $800 \mathrm{kDa}$ and $<400 \mathrm{kDa}$, indicated with arrows), especially when cross-linking in the absence of NEM quenching (Figure 6, lane 4). The bands above the $800 \mathrm{kDa}$ could be non-specific interactions between the $700 \mathrm{kDa}$ complex and other species. It could also be due to the different mobility of the complex caused by crosslinking induced conformational change. While we interpret the $<400 \mathrm{kDa}$ band to be indicative of the G6CV14C variant in the separate Hcf106 pool, which may also form higher oligomers. Overall, these results indicate Hcf106 has a strong tendency to form oligomers and that Hcf106 self-oligomerization might be present in both the receptor complex and the free pool of Hcf106. 


\subsection{Integrated Hcf106 form contacts with both imported cpTatC and precursor proteins}

To validate whether integrated Hcf106 can participate in the transport process directly, we looked for contacts between integrated Hcf106 and imported cpTatC or cpTat pathway precursor proteins. Previous studies found that endogenous Hcf106 can be photo-crosslinked with the signal peptide of precursor proteins when part of a functional receptor complex with cpTatC (Gérard and Cline, 2006), and that integrated, recombinant Hcf106 localizes to a complex that could bind precursor (Cline and Mori, 2001).

Earlier work demonstrated that replacing the original cpTatC transit peptide with the transit peptide of the precursor to the small subunit of RuBisCO resulted in higher efficiency of pre-cpTatC import, mature cpTatC localization to thylakoid that could be detected in direct contact with both the RR proximal region on a Tat pathway precursor signal peptide and Tha4 (Aldridge et al., 2012; Ma and Cline, 2013; Aldridge et al., 2014). To confirm Cys-substituted Hcf106 participation in the receptor complex, we selected five Hcf106 Cys variants from the transmembrane domain and looked to see if they interact with imported cpTatCV270C. We initially chose transmembrane locations for cysteine substitution because recently the cpTatC bacterial homolog, TatC, was found to form crosslinks with the TatB (Hcf106) transmembrane domain via TatC TM5 (Kneuper et al., 2012; Rollauer et al., 2012). Imported cpTatC270C was previously used to map Tha4 binding (Ma and Cline, 2013). We observed $\left[{ }^{3} \mathrm{H}\right] \mathrm{cpTatC}$ at an apparent molecular weight of $\sim 28 \mathrm{kDa}$, which matches the apparent molecular weight for endogenous cpTatC. Samples with integrated Hcf106, containing Cys-substitutions in the hydrophobic core of the Hcf106 transmembrane domain, e.g., A12C, L13C and $\mathrm{V} 14 \mathrm{C}$, formed interactions with cpTatCV270C, showing a $\sim 56 \mathrm{kDa}$ adduct that is not found in the control lane (Figure 7A). For the control lane, no Hcf106 was integrated into the thylakoid. In contrast, L20C, which is closer to the stromal side of the membrane, showed a similar result as the control lane. The data here demonstrate that integrated Hcf106 is in close contact with cpTatC.

Alternatively, to confirm that integrated, recombinant, Cys-substituted Hcf106 was able to bind precursor, we integrated wild type Hcf106 or various Cys-substituted Hcf106 into thylakoid and used these membranes in precursor binding assays. The precursor, tOE17-25C/V-20F, was shown to bind tightly to the first cpTatC stromal loop (Gérard and Cline, 2007) and was used previously cpTatC crosslinking assays (Ma and Cline, 2010). It is a modified precursor of the $17 \mathrm{kDa}$ subunit of the oxygen evolving complex, containing a truncated signal peptide (tOE17), a cysteine inserted on the $\mathrm{N}$ terminal side of the twin arginine motif, 25 amino acids from the signal peptide cleavage site (-25C), and a phenylalanine substituted for the valine at position -20 from the signal peptide cleavage site (V-20F) [Figure 7, see reference (Ma and Cline, 2010). We subjected tOE17-25C/V-20F to cross-linking with the Hcf106 C-terminal APH region as it interacts with cpTatC stromal loop 1 (Figure $7 \mathrm{C}$ ). This loop of cpTatC was also identified as interacting with tOE17-25C/V-20F. tOE17-25C/V-20F showed an interaction with Hcf106T38C, E48C, R54C, E63C, and I69C by the presence of a higher molecular weight adduct when analyzed by non-reducing SDS-PAGE. The strongest interactions involved Hcf106R54C, E63C, and I69C (Figure 7C, lanes 3-7), while wild type Hcf106, lacking cysteine, was not able to generate an adduct (Figure 7C, lane 2). 
Taken together these data suggest that Hcf106 variants are incorporating into receptor complexes and can play a functional role in precursor binding.

\section{Discussion}

Recently, structural insights of the individual prokaryotic Tat components were revealed which lay a solid foundation for deciphering the mechanism of the Tat system (Hu et al., 2010; Rollauer et al., 2012; Ramasamy et al., 2013; Zhang et al., 2014). Additionally Blümmel et al. used photo-crosslinking to elucidate the architecture of TatBC oligomers during the initial assembly step, but due to the nature of the technique could only provide specific residue location for one binding partner (Blummel et al., 2015). Here, cysteine scanning by disulfide bond formation was used to provide a precise map of interactions between the two homologous proteins in thylakoid. Previous work using isolated thylakoids demonstrated a substrate-gated docking of Tha4 into the срTatC cavity initiating translocase assembly (Aldridge et al., 2014) and challenging the receptor complex model from E.coli in which TatB forms a ring-like structure in the center with TatC occupying the peripheral positions (Maurer et al., 2010; Cline, 2015). However, how Hcf106-cpTatC are arranged to accommodate Tha4 docking and whether there are organizational differences between TatBC in E. coli and Hcf106cpTatC in thylakoid have not been determined yet due to a lack of methodology to study the role of Hcf106 in isolate membranes.

Despite sharing significant sequence similarity, Tha4 and Hcf106 have distinct roles in protein transport (Cline and Mori, 2001; Dabney-Smith et al., 2003). Unlike Tha4, Hcf106 is typically found tightly complexed with cpTatC. Therefore, the use of the a-Hcf106 antibody is not viable as a sequestrant for native Hcf106 as it was for Tha4 (Dabney-Smith et al., 2003) due to the tight association of Hcf106 with cpTatC. Previous experiments showed that integration of in vitro translated $\left[{ }^{3} \mathrm{H}\right] \mathrm{Hcf} 106$ into thylakoid assembled into a $700 \mathrm{kDa}$ receptor complex as analyzed by BN-PAGE (Fincher et al., 2003), setting the stage for a possible involvement of exogenously Hcf106 in the receptor complex. Here we further characterize integration of in vitro expressed Hcf106 using cysteine substitutions and disulfide bond formation to demonstrate selfinteractions and interactions with imported $\mathrm{cpTatC}$ and precursor. Introduction of cysteine substitutions into Hcf106 largely did not affect integration into the membrane or presence in the $700 \mathrm{kDa}$ complex. Exceptions include Cys-substitutions in areas such as the TMD hydrophobic core, which lowered integration overall, likely due to a decrease in helix hydrophobicity, and in the hinge region, which may also be buried or involved in contacts with cpTatC. However, most of the Hcf106 Cys-substitutions tested do integrate into thylakoid allowing the study of the organization of the protein in a native membrane. In E. coli, in the absence of TatC, TatB formed a ladder of bands of about $100 \mathrm{kDa}$ to over $880 \mathrm{kDa}$, suggesting that TatB has oligomeric properties on its own when it is not associated with TatC (Behrendt et al., 2007; Cleon et al., 2015). In the present study, we see ladders of full length and truncated Hcf106, which may be indicative of the separate pool of Hcf106; however, we also see formation of ladders of cpTatC that correspond in a linear manner to the integration of the truncated version of Hcf106. We conclude, therefore, that a substantial fraction of the incorporated, truncated Hcf106 is a part of the receptor complex with endogenous cpTatC. 
We also identified residues in Hcf106 that are important to receptor complex assembly. For example, in the hinge region, e.g., G24C G27C, Cys-substituted Hcf106 lost its ability to assemble into a $700 \mathrm{kDa}$ receptor complex as analyzed by BN-PAGE, suggesting important contacts have been disrupted. This is in agreement with observations in $E$. coli TatB hinge where the Gly/Pro residues have been identified as essential for efficient substrate export (Barrett et al., 2003). There are at least three possible explanations for the absence of the $700 \mathrm{kDa}$ complex when cysteines are substituted into the hinge of Hcf106. The first is that the substitution with Cys at those residues inhibited Hcf106-Hcf106 or Hcf106-cpTatC interactions, resulting in the absence of the $700 \mathrm{kDa}$ complex. Second, Cys substitutions at those residues did not inhibit assembly per se but impacted the stability of the $700 \mathrm{kDa}$ complex resulting in a receptor complex to be unable to withstand digitonin solubilization. Third, these substitutions resulted in decreased integration and therefore decreased participation in the $700 \mathrm{kDa}$ complex. The third explanation is unlikely because Cys substitutions in the hinge region did not appear to inhibit integration and resistance to alkaline extraction of Hcf106. However, the data do suggest that the $\mathrm{N}$ terminus around residues $\mathrm{G} 8$ and $\mathrm{E} 11$ and the hinge region around residues G24 and G27 are of great structural significance to the assembly or stability of the interaction between Hcf106 and cpTatC. Glycine often introduces more flexibility to protein structure, and so in replacing glycine with cysteine, the overall flexibility of the hinge region might be changed to interfere with an interaction with срTatC. In addition to glycine, residue charge has been known to play an important role in membrane protein stability, possibly by forming a salt bridge and oligomeric structure solubilization (Wimley et al., 1996). For example, Tha4 contains a glutamate (E10) in the transmembrane region that has been shown to be critical for Tha4 function, possibly by stabilization of oligomers through salt bridge formation (Dabney-Smith et al., 2003). However, whether E11 in Hcf106 has a similar role is unclear. Other Hcf106 residues, such as E41, Q43, and P44 may also affect interaction with cpTatC as shown by a decrease in the presence of those Hcf106 Cys variants in the $700 \mathrm{kDa}$ complex. By replacing residues with Cys, Hcf106 contacts with cpTatC would be altered. Further studies are needed to clarify which region of $\mathrm{cpTatC}$ interacts with the $\mathrm{N}$-terminus and hinge region of Hcf106. Currently, due to the existence of endogenous Hcf106, we are unable to determine whether these cysteine variants affect receptor complex functionality.

Truncated Hcf106 1 107 can assemble with endogenous cpTatC indicating that the C-tail is less important as compared with the TMD and APH for receptor complex formation. This is also in agreement with the observation in E.coli that significant transport was observed when 70 residues were removed from C-terminus of TatB (Lee et al., 2002). We designed these experiments to maximize the observable amount of the $700 \mathrm{kDa}$ receptor complex relative to homo-oligomeric Hcf106 complexes by adjusting the detergent:chlorophyll ratio. Based on our earlier BN-PAGE data, the homooligomeric forms should not appear on the gel. This allows us to interpret the lower bands seen when the truncated Hcf106 is incorporated into the receptor complex. As the amount of in vitro translated $\left[{ }^{3} \mathrm{H}\right] \mathrm{Hcf} 106_{1-107}$ increases, more of the truncated protein assembled into a complex of lower molecular weight with $\mathrm{cpTatC}$, indicating that Hcf106 $_{1-107}$ has the ability to compete with endogenous Hcf106 and as the concentration of Hcf106 $1-107$ increases, more cpTatC assembled with the truncated Hcf106. However, 
based on the immunoblotting data, the proportion of full length Hcf106 in a $700 \mathrm{kDa}$ complex is less. The apparent $\sim 600 \mathrm{kDa}$ and $\sim 500 \mathrm{kDa}$ bands, together with the double Cys cross-linking data, strongly suggest that integrated recombinant Hcf106 may form oligomers in a separate pool and may compete with the endogenous Hcf106 for association with cpTatC. If the receptor complex contains eight copies of both Hcf106 and cpTatC as predicted (Mori et al., 2001; Celedon and Cline, 2012), the $\sim 600 \mathrm{kDa}$ complexes may indicate approximately four endogenous Hcf106 were replaced with the truncated version while the $\sim 500 \mathrm{kDa}$ complexes indicates most, if not all, of the endogenous Hcf106 were replaced by truncated Hcf106.

Hcf106 shares structural similarity with Tha4. Both biochemical labeling (Aldridge et al., 2012) of Tha4 as well as solution NMR and computer simulation modeling (Rodriguez et al., 2013) of E. coli homolog TatA show that the TMD is tilted and the Nterminal APH is partially embedded in the membrane, rather than the TMD inserted vertically (e.g., TMD parallel to the bilayer normal) and the APH laying on the surface of membrane (e.g., perpendicular to the bilayer normal). The cross-linking data of Hcf106 presented here also suggest a similar topology for Hcf106. We saw limited interactions between residues L28C to R40C (N-proximal region of the APH), which could be explained by being in the low dielectric environment of the hydrophobic core of the membrane.

In summary, to systematically study Hcf106, a critical component in the chloroplast Tat system, a recombinant library of single Cys-substitutions from the Nterminus to the end of APH was generated. Here we demonstrate that exogenous, recombinant Hcf106 was able to insert into thylakoid and participates directly in the cpTat receptor complex, likely by replacing endogenous, Cys-less versions of Hcf106. This library not only helped clarify Hcf106 self-contacts but also demonstrated that exogenously integrated Hcf106 interacts with the precursor signal peptide via the Hcf106 APH and that the TMD was identified to form close contacts with cpTatC TM5. These interaction data further confirm the capability of in vitro integrated Hcf106 to function in the thylakoid membrane system and demonstrates a new tool to evaluate the organization of cpTat complexes.

\section{Acknowledgements}

580 Science (Award DE-SC0014441 to CDS). The authors wish to thank Ken Cline

581 (University of Florida) for the cpTatC clones and members of the Dabney-Smith lab for 582 critical reading of the manuscript.

583

584

\section{Author Contributions}

586

QM and CDS designed the research; QM and KF performed research; QM, KF, CPN, and CDS analyzed data; QM, CPN, and CDS wrote the manuscript. 
References

Alami M, Luke I, Deitermann S, Eisner G, Koch HG, Brunner J, Müller M (2003) Differential interactions between a twin-arginine signal peptide and its translocase in Escherichia coli. Mol Cell 12: 937-946

Aldridge C, Ma X, Gerard F, Cline K (2014) Substrate-gated docking of pore subunit Tha4 in the TatC cavity initiates Tat translocase assembly. The Journal of cell biology 205: 51-65

Aldridge C, Ma X, Gerard F, Cline K (2014) Substrate-gated docking of pore subunit Tha4 in the TatC cavity initiates Tat translocase assembly. J Cell Biol 205: 51-65

Aldridge C, Storm A, Cline K, Dabney-Smith C (2012) The chloroplast twin arginine transport (tat) component, tha4, undergoes conformational changes leading to tat protein transport. J Biol Chem 287: 34752-34763

600

Barrett CM, Mathers JE, Robinson C (2003) Identification of key regions within the Escherichia coli TatAB subunits. FEBS Lett 537: 42-46

Behrendt J, Bruser T (2014) The TatBC complex of the Tat protein translocase in Escherichia coli and its transition to the substrate-bound TatABC complex. Biochemistry 53: 2344-2354

Behrendt J, Lindenstrauss U, Bruser T (2007) The TatBC complex formation suppresses a modular TatB-multimerization in Escherichia coli. FEBS Lett 581: 4085-4090

Berks BC (2015) The twin-arginine protein translocation pathway. Annual review of biochemistry 84: 843-864

Blummel AS, Haag LA, Eimer E, Muller M, Frobel J (2015) Initial assembly steps of a translocase for folded proteins. Nat Commun 6: 7234

Bolhuis A, Mathers JE, Thomas JD, Barrett CM, Robinson C (2001) TatB and TatC form a functional and structural unit of the twin- arginine translocase from Escherichia coli. J Biol Chem 276: 20213-20219.

Braun NA, Davis AW, Theg SM (2007) The chloroplast Tat pathway utilizes the transmembrane electric potential as an energy source. Biophys J 93: 1993-1998

Celedon JM, Cline K (2012) Stoichiometry for binding and transport by the twin arginine translocation system. The Journal of cell biology 197: 523-534

Celedon JM, Cline K (2013) Intra-plastid protein trafficking: How plant cells adapted prokaryotic mechanisms to the eukaryotic condition. Biochimica Et Biophysica Acta-Molecular Cell Research 1833: 341-351

Cleon F, Habersetzer J, Alcock F, Kneuper H, Stansfeld PJ, Basit H, Wallace MI, Berks BC, Palmer T (2015) The TatC component of the twin-arginine protein translocase functions as an obligate oligomer. Molecular microbiology

Cline K (1986) Import of proteins into chloroplasts. Membrane integration of a thylakoid precursor protein reconstituted in chloroplast lysates. J Biol Chem 261: 1480414810

Cline K (2015) Mechanistic Aspects of Folded Protein Transport by the Twin Arginine Translocase (Tat). J Biol Chem 290: 16530-16538

Cline K (2015) Mechanistic Aspects of Folded Protein Transport by the Twin Arginine Translocase (Tat). The Journal of biological chemistry 290: 16530-16538

Cline K, Henry R, Li C, Yuan J (1993) Multiple pathways for protein transport into or across the thylakoid membrane. Embo J 12: 4105-4114 
Cline K, Mori H (2001) Thylakoid DeltapH-dependent precursor proteins bind to a cpTatC-Hcf106 complex before Tha4-dependent transport. J Cell Biol 154: 719729.

Dabney-Smith C, Cline K (2009) Clustering of C-terminal stromal domains of Tha4 homo-oligomers during translocation by the Tat protein transport system. Mol Biol Cell 20: 2060-2069

Dabney-Smith C, Mori H, Cline K (2003) Requirement of a Tha4-conserved transmembrane glutamate in thylakoid Tat translocase assembly revealed by biochemical complementation. J Biol Chem 278: 43027-43033

Dabney-Smith C, Mori H, Cline K (2006) Oligomers of Tha4 organize at the thylakoid Tat translocase during protein transport. J Biol Chem 281: 5476-5483

Fincher V, Dabney-Smith C, Cline K (2003) Functional assembly of thylakoid deltapHdependent/Tat protein transport pathway components in vitro. Eur J Biochem 270: $4930-4941$

Gérard F, Cline K (2006) Efficient twin arginine translocation (Tat) pathway transport of a precursor protein covalently anchored to its initial cpTatC binding site. J Biol Chem 281: 6130-6135

Gérard F, Cline K (2007) The thylakoid proton gradient promotes an advanced stage of signal peptide binding deep within the Tat pathway receptor complex. J Biol Chem 282: 5263-5272

Holzapfel E, Eisner G, Alami M, Barrett CM, Buchanan G, Luke I, Betton JM, Robinson C, Palmer T, Moser M, Muller M (2007) The entire N-terminal half of TatC is involved in twin-arginine precursor binding. Biochemistry 46: 2892-2898

Hu Y, Zhao E, Li H, Xia B, Jin C (2010) Solution NMR structure of the TatA component of the twin-arginine protein transport system from gram-positive bacterium Bacillus subtilis. J Am Chem Soc 132: 15942-15944

Kneuper H, Maldonado B, Jager F, Krehenbrink M, Buchanan G, Keller R, Muller M, Berks BC, Palmer T (2012) Molecular dissection of TatC defines critical regions essential for protein transport and a TatB-TatC contact site. Molecular microbiology 85: 945-961

Lee PA, Buchanan G, Stanley NR, Berks BC, Palmer T (2002) Truncation analysis of Tat $A$ and $T a t B$ defines the minimal functional units required for protein translocation. J Bacteriol 184: 5871-5879

Lee PA, Orriss GL, Buchanan G, Greene NP, Bond PJ, Punginelli C, Jack RL, Sansom MS, Berks BC, Palmer T (2006) Cysteine-scanning mutagenesis and disulfide mapping studies of the conserved domain of the twin-arginine translocase TatB component. J Biol Chem 281: 34072-34085

Ma X, Cline K (2010) Multiple precursor proteins bind individual Tat receptor complexes and are collectively transported. EMBO J 29: 1477-1488

Ma X, Cline K (2013) Mapping the signal peptide binding and oligomer contact sites of the core subunit of the pea twin arginine protein translocase. The Plant cell 25: 999-1015

Maldonado B, Kneuper H, Buchanan G, Hatzixanthis K, Sargent F, Berks BC, Palmer T (2011) Characterisation of the membrane-extrinsic domain of the TatB component of the twin arginine protein translocase. FEBS letters 585: 478-484 
Maurer C, Panahandeh S, Jungkamp AC, Moser M, Müller M (2010) TatB functions as an oligomeric binding site for folded Tat precursor proteins. Mol Biol Cell 21: 4151-4161

Mori H, Cline K (2002) A twin arginine signal peptide and the $\mathrm{pH}$ gradient trigger reversible assembly of the thylakoid [Delta]pH/Tat translocase. J Cell Biol 157: 205-210.

Mori H, Summer EJ, Cline K (2001) Chloroplast TatC plays a direct role in thylakoid (Delta)pH-dependent protein transport. FEBS Lett 501: 65-68.

Pal D, Fite K, Dabney-Smith C (2013) Direct interaction between a precursor mature domain and transport component Tha4 during twin arginine transport of chloroplasts. Plant physiology 161: 990-1001

Pettersson P, Ye W, Jakob M, Tannert F, Klosgen RB, Maler L (2018) Structure and dynamics of plant TatA in micelles and lipid bilayers studied by solution NMR. FEBS J 285: 1886-1906

Ramasamy S, Abrol R, Suloway CJ, Clemons WM, Jr. (2013) The glove-like structure of the conserved membrane protein TatC provides insight into signal sequence recognition in twin-arginine translocation. Structure 21: 777-788

Rodriguez F, Rouse SL, Tait CE, Harmer J, De RA, Timmel CR, Sansom MS, Berks BC, Schnell JR (2013) Structural model for the protein-translocating element of the twin-arginine transport system. Proceedings of the National Academy of Sciences of the United States of America 110: E1092-1101

Rolland N, Ferro M, Ephritikhine G, Marmagne A, Ramus C, Brugiere S, Salvi D, Seigneurin-Berny D, Bourguignon J, Barbier-Brygoo H, Joyard J, Garin J (2006) A versatile method for deciphering plant membrane proteomes. J Exp Bot 57: 1579-1589

Rollauer SE, Tarry MJ, Graham JE, Jaaskelainen M, Jager F, Johnson S, Krehenbrink M, Liu SM, Lukey MJ, Marcoux J, McDowell MA, Rodriguez F, Roversi P, Stansfeld PJ, Robinson CV, Sansom MS, Palmer T, Hogbom M, Berks BC, Lea SM (2012) Structure of the TatC core of the twin-arginine protein transport system. Nature 492: 210-214

Schneider CA, Rasband WS, Eliceiri KW (2012) NIH Image to ImageJ: 25 years of image analysis. Nat Methods 9: 671-675

Wimley WC, Gawrisch K, Creamer TP, White SH (1996) Direct measurement of saltbridge solvation energies using a peptide model system: implications for protein stability. Proceedings of the National Academy of Sciences of the United States of America 93: 2985-2990

Zhang Y, Wang L, Hu Y, Jin C (2014) Solution structure of the TatB component of the twin-arginine translocation system. Biochimica et biophysica acta 1838: 18811888

\section{Figure Legends}

Figure 1. Topologic model of Hcf106 based on experimental and predictive structural features. Primary residue sequence of Hcf106 from Pisum sativum. Amino acids in regions under investigation in this study are numbered (2-71). Dark gray indicates the hydrophobic core (i.e. acyl chains) of the membrane, while the lighter gray indicates the lipid head groups. 
Figure 2. Blue-native gel analysis of the integration of recombinant Hcf106 cysteine variants. $\left[{ }^{3} \mathrm{H}\right] \mathrm{Hcf} 106$ single cysteine variants containing complexes were analyzed by BN-PAGE and fluorography (Materials and Methods). Single Cys substitutions at multiple positions in Hcf106 are indicated across the top of the panels. The $700 \mathrm{kDa}$ complex is indicated by an arrow. Wild-type Hcf106 was used as a control. Molecular mass markers are ferritin (880 and $440 \mathrm{kDa}$ ) and BSA (132 kDa). Gels are representative of at least three experiments.

\section{Figure 3. Truncated Hcf106 1-107 assembles into a complex with endogenous} cpTatC. An increasing concentration of Hcf106 1-107 was integrated into thylakoid and subjected to digitonin solubilization, BN-PAGE, and analysis by fluorography (left panel) as in Figure 2. To detect whether this truncated form can generate a different size of the receptor complex, samples were also subjected to immunoblotting with anti-cpTatC (middle panel), anti-Hcf106 (right panel) antibodies. The amount of Hcf1061-107 added to thylakoid corresponds to dilutions of the in vitro translation reaction (i.e., 1:60, 1:12, 1:6, and 1:3). Gels are representative of at least three separate experiments. As Hcf1061-107 (asterisks).

Figure 4. Hcf106 forms a dimer when cysteine substitutions are in $\mathrm{N}$-terminus, the central TMD, and the APH regions. In vitro translated Hcf106 variants were integrated into NEM pre-treated thylakoid membranes and subjected to oxidizing conditions (1 mM CuP) as described (Materials and Methods). Samples were resolved by SDS-PAGE and protein bands were visualized by fluorography. The amino acid position of each cysteine substitution is shown above each panel. Hcf106 monomer (m) and dimer (di) forms are indicated at the right of the panels. Gels are representative of at least three separate experiments.

Figure 5. Helical wheel projection of the TMD and APH regions of Hcf106 reveals periodic interactions. Helical wheel projections were generated using the Protean module of DNAStar (Lasergene, Madison WI). (A) The TMD and Hinge of Hcf106, residues A12C-G27C; (B) the N-terminal proximal portion of the APH of Hcf106, residues L28C-P44C; and (C) the C-terminal proximal portion of the APH of Hcf106, residues T45C-G65C. The shading represents the hydrophobicity of the amino acid, hydrophilic amino acids are more lightly shaded. Stars indicate the presence of a dimer that is $>10 \%$ of total (see Supplemental Figure S2).

764

765

766

767

Figure 6. Double cysteine substitutions demonstrate a higher order Hcf106 complex. (A) Lanes 1-6 show the cross-linking of double cysteine variants L13CL21C, 149CE61C V14CL20C and R54CL60C and the single Cys variant L21C under nonreducing conditions. Lanes 7-12 show the same samples under reducing conditions. The amount of protein integrated relative to the parent single Cys variant, $\mathrm{L} 21 \mathrm{C}$, is shown below lanes 7-12. Translation products diluted 1:40 are shown in lanes 13-17.

768 Molecular weight markers are indicated on the right and left. (B) Crosslinking with the

769 G6CV14C double cysteine variant was compared to the single Cys mutant V14C and 
double mutant V14CL20C (lanes 1-3). G6CV14C showed higher ordered Hcf106 complexes up to octamers when analyzed by 10-20\% SDS-PAGE (lane 4). (C) Crosslinked G6CV14C double Cys variant has reduced capability to incorporate into the receptor complex. Lane 2 is the standard cross-linking condition with 5 min incubation of CuP and the reaction was quenched with $50 \mathrm{mM}$ NEM. Lane 1: translation product was pre-treated with NEM and subjected to standard cross-linking. Lanes 3-6: cross-linking under variable time or with/without NEM quenching. Lane 6: WT Hcf106 under standard cross-linking conditions. The same samples from lanes 1-5 were also subjected to nondenaturing SDS-PAGE (lane 7-11). Asterisks indicate the number of Hcf106 monomers in the oligomer as determined by size. Arrows indicate additional bands of cross-linked G6CV14C. The amount of protein integrated relative to the parent single Cys variants, $\mathrm{V} 14 \mathrm{C}$, is shown below the lanes. Gels in both panels represent at least three separate experiments.

Figure 7. Integrated Hcf106 interacts directly with imported cpTatC and the signal peptide of the precursor. (A) In vitro translated $\left[{ }^{3} \mathrm{H}\right] \mathrm{cp} T a t C a a a V 270 \mathrm{C}$ was imported into intact chloroplasts. Intact chloroplasts were isolated over a 35\% Percoll cushion (Materials and Methods) and recovered thylakoid were used for integration of unlabeled Hcf106 Cys variants, as indicated across the top of the panels, or the same volume of $\mathrm{IB}, 10 \mathrm{mM} \mathrm{MgCl} 2$ as control. Hcf106 TMD shows cross-linking with cpTatC TM5 in a $\sim 56 \mathrm{kDa}$ band that migrates higher than the nonspecific bands in the control lane.

791 Translation products (tp) diluted 1:40 are shown. (B) Residue sequence of the signal peptide of tOE17-25CV-20F showing the -25C substitution, the RR motif, and the V-20F substitution. (C) In vitro translated unlabeled Hcf106 Cys variants, as indicated across the top of the panel, were integrated into isolated thylakoids. Thylakoid were then washed to remove unintegrated protein (Materials and Methods) and incubated with either precursor, $\left[{ }^{3} \mathrm{H}\right] \mathrm{tOE} 17-25 \mathrm{CV}-20 \mathrm{~F}$, or the same volume of $\mathrm{IB}, 10 \mathrm{mM} \mathrm{MgCl}$ as control. Gels in both panels are representative of at least three separate experiments. 
Ma, et al., Figure 1

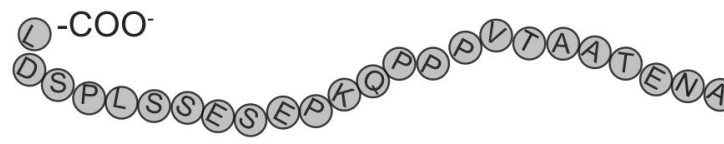

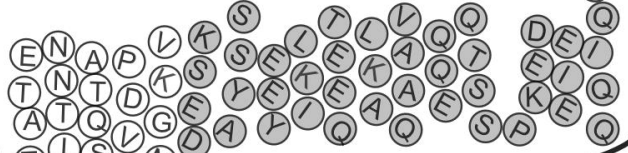

Q(1)

(1) $P$

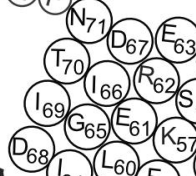

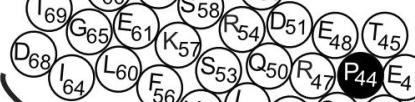

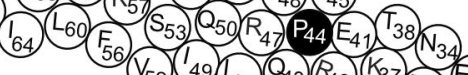

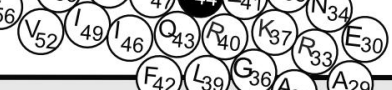

Stroma

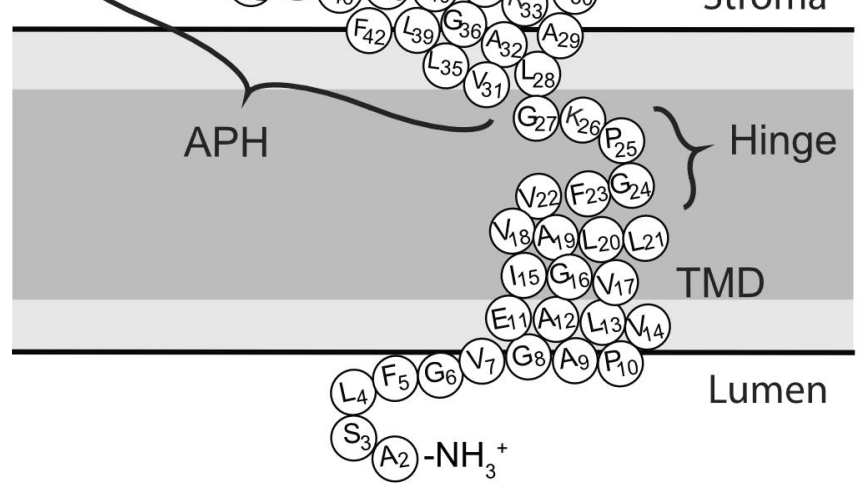


Ma, et al., Figure 2 $800-$

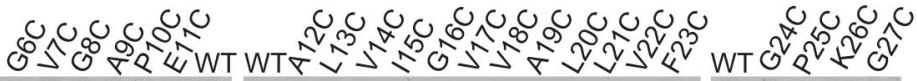

$\overrightarrow{400}$

$132-$

$\mathrm{kDa}$

1234567

$\llcorner\mathrm{N}$-term -

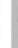

ก-

(1) 


\section{Ma, et al., Figure 3}

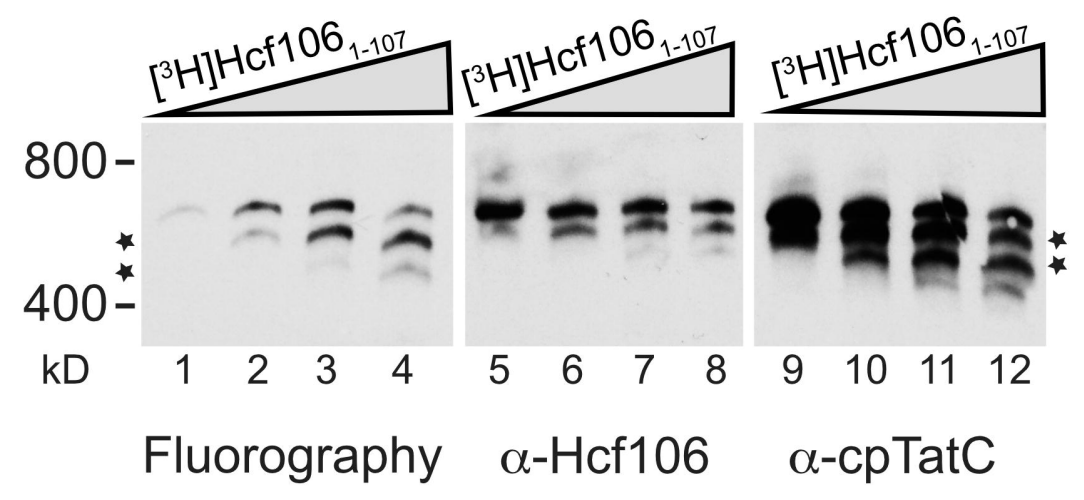


Ma, et al., Figure 4
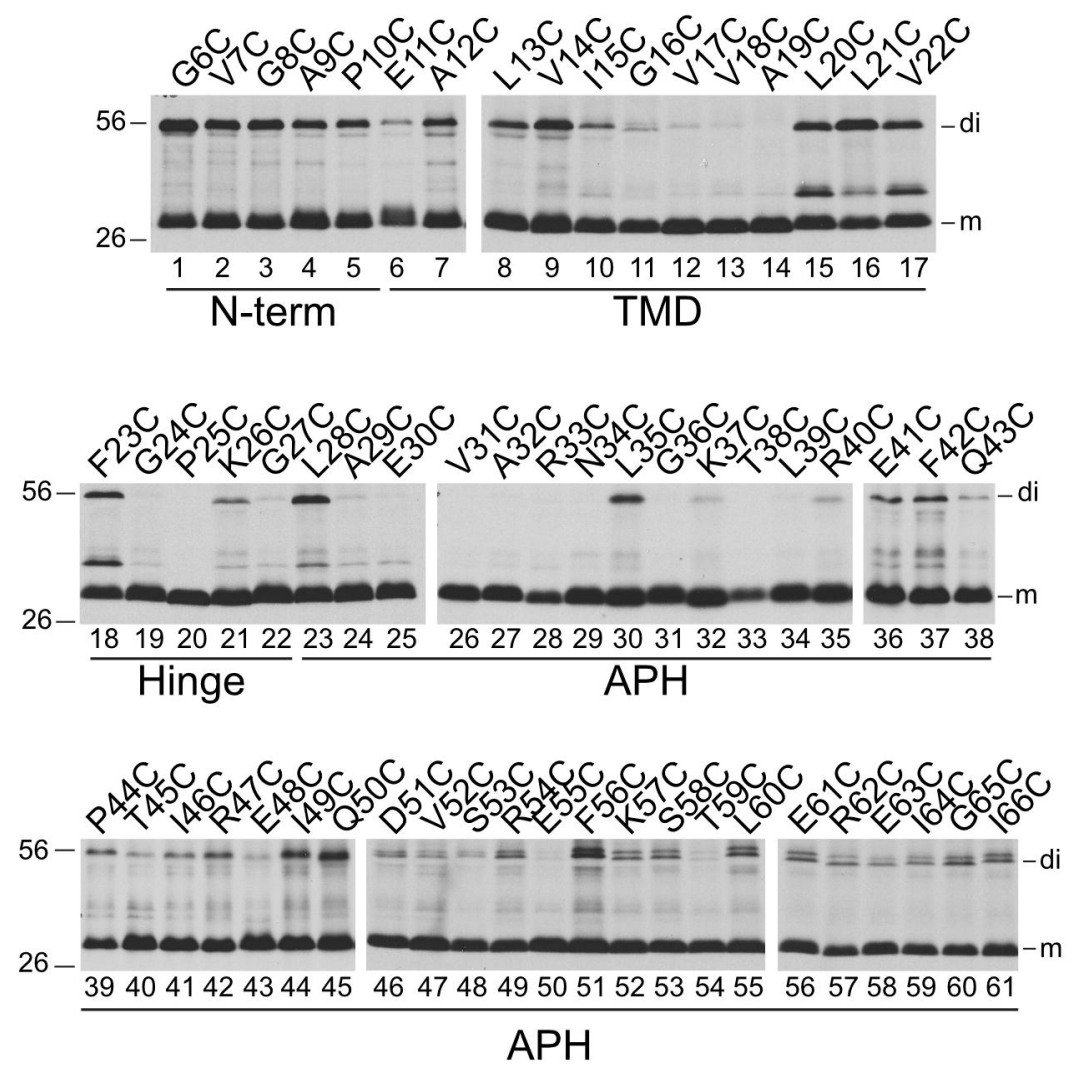
Ma, et al., Figure 5

A.

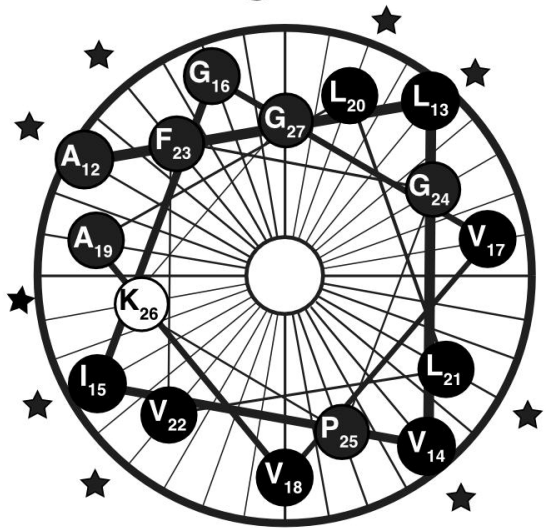

TMD + hinge (A12-G27)

B.

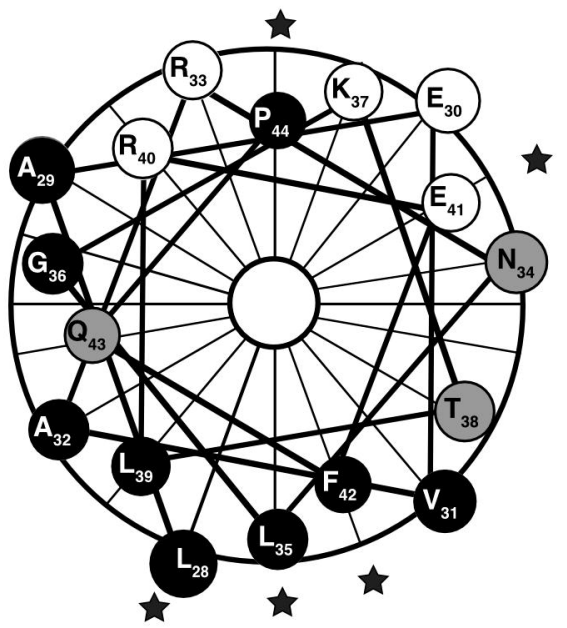

APH (L28-P44)

C.

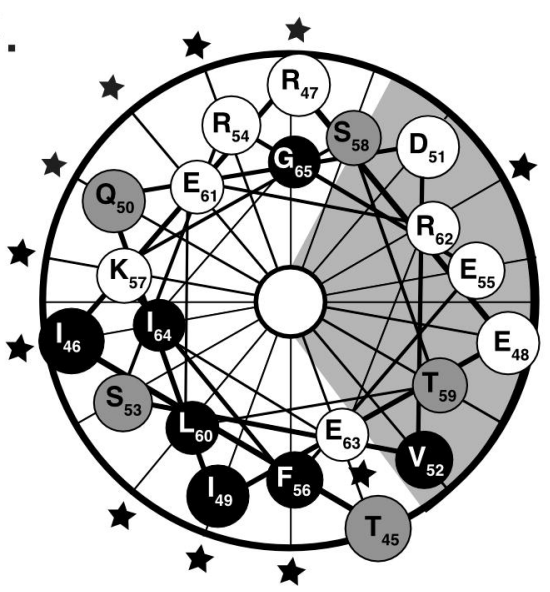

APH (T45-G65) 


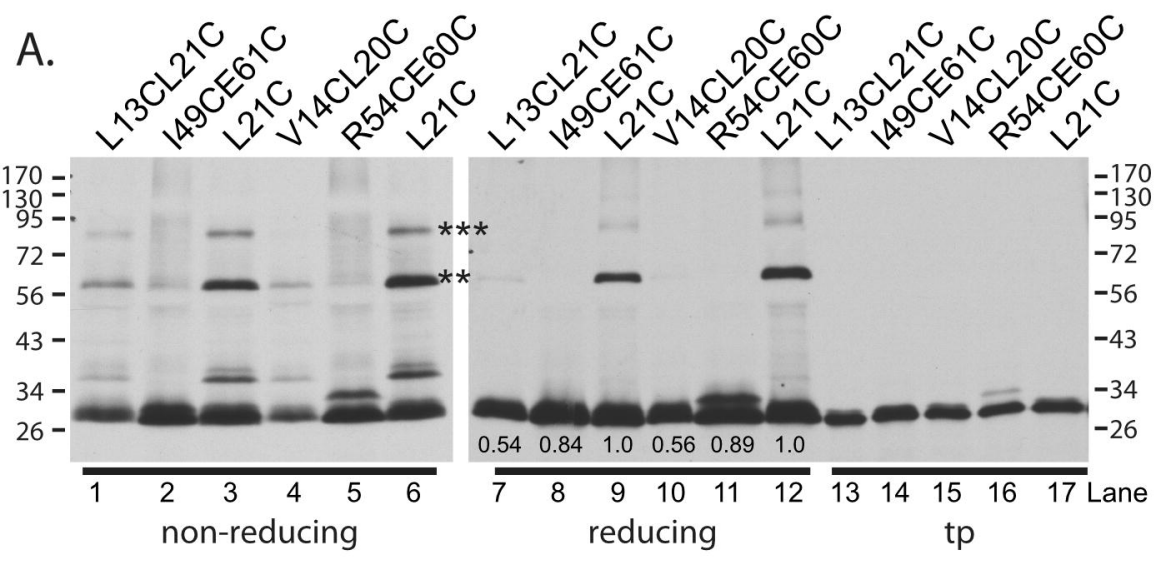

B.

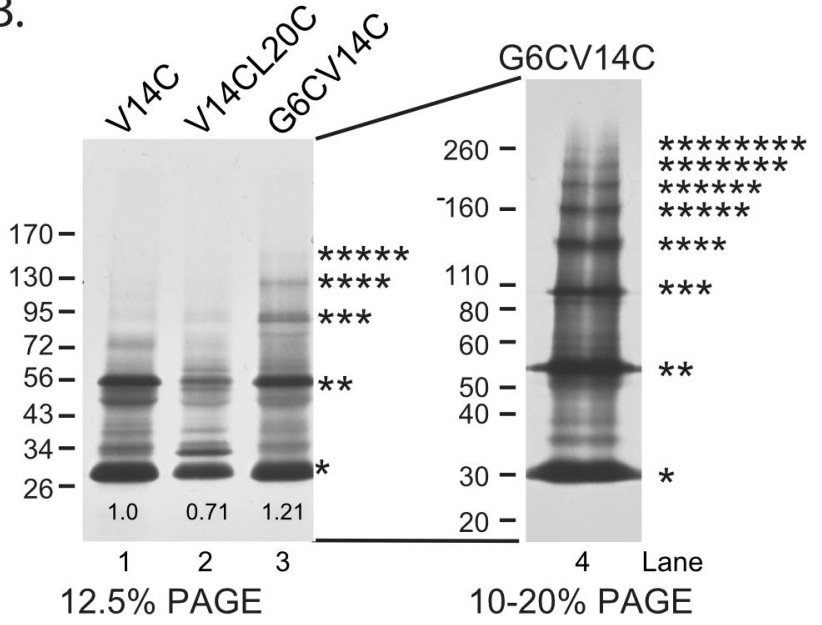

c.

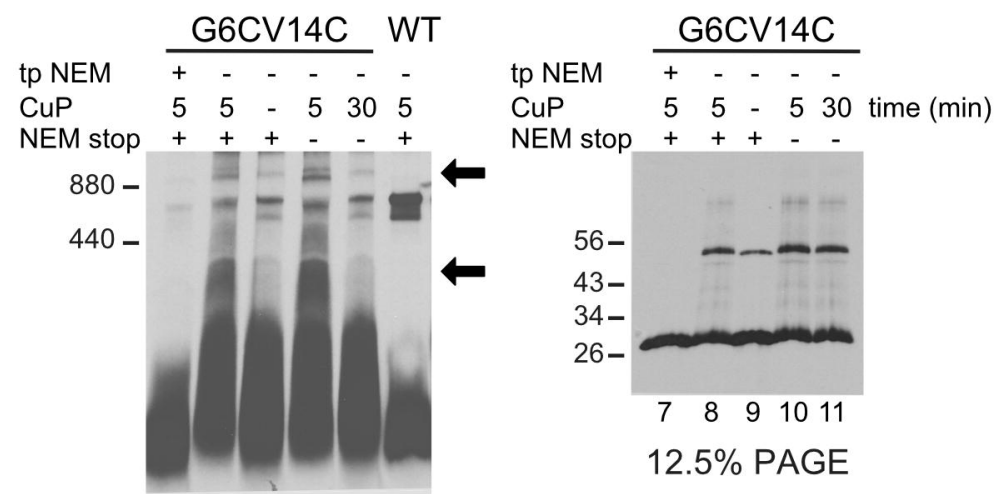


